\title{
Stability and estimate of solution to uncertain neutral delay systems
}

\author{
Alexander Domoshnitsky ${ }^{1 *}$, Michael Gitman ${ }^{2}$ and Roman Shklyar ${ }^{1}$
}

\author{
"Correspondence: adom@ariel.ac.il \\ 1 Department of Mathematics and \\ Computer Science, Ariel University, \\ Ariel, Israel \\ Full list of author information is \\ available at the end of the article
}

\begin{abstract}
The coefficients and delays in models describing various processes are usually obtained as a results of measurements and can be obtained only approximately. We deal with the question of how to estimate the influence of 'mistakes' in coefficients and delays on solutions' behavior of the delay differential neutral system $x_{i}^{\prime}(t)-q_{i}(t) x_{i}^{\prime}\left(t-\theta_{i}(t)\right)+\sum_{j=1}^{n}\left(p_{i j}(t)-\Delta p_{i j}(t)\right) x_{j}\left(t-\tau_{i j}(t)-\Delta \tau_{i j}(t)\right)=f_{i}(t), i=1, \ldots, n$, $t \in[0, \infty)$. This topic is known in the literature as uncertain systems or systems with interval defined coefficients. The goal of this paper is to obtain stability of uncertain systems and to estimate the difference between solutions of a 'real' system with uncertain coefficients and/or delays and corresponding 'model' system. We develop the so-called Azbelev W-transform, which is a sort of the right regularization allowing researchers to reduce analysis of boundary value problems to study of systems of functional equations in the space of measurable essentially bounded functions. In corresponding cases estimates of norms of auxiliary linear operators (obtained as a result of $W$-transform) lead researchers to conclusions about existence, uniqueness, positivity and stability of solutions of given boundary value problems. This method works efficiently in the case when a 'model' used in $W$-transform is 'close' to a given 'real' system. In this paper we choose, as the 'models', systems for which we know estimates of the resolvent Cauchy operators. We demonstrate that systems with positive Cauchy matrices present a class of convenient 'models'. We use the $W$-transform and other methods of the general theory of functional differential equations. Positivity of the Cauchy operators is studied and then used in the analysis of stability and estimates of solutions.

Results: We propose results about exponential stability of the given system and obtain estimates of difference between the solution of this uncertain system and the 'model' system $x_{i}^{\prime}(t)-q_{i}(t) x_{i}^{\prime}\left(t-\theta_{i}(t)\right)+\sum_{j=1}^{n} p_{i j}(t) x_{j}\left(t-\tau_{i j}(t)\right)=f_{i}(t), i=1, \ldots, n, t \in[0, \infty)$. New tests of stability and in the future of existence and uniqueness of boundary value problems for neutral delay systems can be obtained on the basis of this technique.
\end{abstract}

Keywords: neutral delay systems; Cauchy matrix; W-method; positivity of the Cauchy matrix; estimates of solutions

\section{Introduction}

In systems of differential equations, modeling real processes, the coefficients and delays are usually known only approximately. For example, models in economics are obtained as a result of corresponding long time observation and regression procedures (see, for example, [1]). The coefficients and delays in model describing various processes in physics are usually obtained as a results of measurements and also can be obtained only approximately [2]. The same situation can be noted in models of population dynamics [3]. A natural

\section{算 Springer}

(92014 Domoshnitsky et al.; licensee Springer. This is an Open Access article distributed under the terms of the Creative Commons Attribution License (http://creativecommons.org/licenses/by/2.0), which permits unrestricted use, distribution, and reproduction in any medium, provided the original work is properly cited. 
question is to estimate the influence of 'mistakes' in coefficients and delays on solutions' behavior of delay differential systems. This topic is known in the literature as uncertain systems or systems with interval defined coefficients.

Important applications present the main reason of attention of many authors to the topic. Systems with uncertain coefficients were considered in the papers [4-22].

Stability of systems with uncertain coefficients was studied in the papers $[6-8,11-14$, $16-18,23]$. Stabilization of uncertain systems with unknown delay was studied in [4, 19, $22]$. Stability of systems with uncertain delay is studied in [12, 15, 20,21]. Stability of neutral uncertain systems was considered in $[10,16,17]$. The basic technique of these publications is the use of Lyapunov-Krasovskii functionals.

Consider the system of the neutral functional differential equations

$$
\begin{aligned}
& x_{i}^{\prime}(t)-\left(S_{i} x_{i}^{\prime}\right)(t)+\sum_{j=1}^{n}\left(p_{i j}(t)-\Delta p_{i j}(t)\right) x_{j}\left(t-\tau_{i j}(t)-\Delta \tau_{i j}(t)\right)=f_{i}(t), \\
& \quad i=1, \ldots, n, t \in[0, \infty),
\end{aligned}
$$

with uncertain coefficients $p_{i j}(t)$ and delays $\tau_{i j}(t)$ and the model system

$$
x_{i}^{\prime}(t)-\left(S_{i} x_{i}^{\prime}\right)(t)+\sum_{j=1}^{n} p_{i j}(t) x_{j}\left(t-\tau_{i j}(t)\right)=f_{i}(t), \quad i=1, \ldots, n, t \in[0, \infty),
$$

where

$$
x_{i}(\xi)=0, \quad i=1, \ldots, n, \xi<0 .
$$

We assume here that $p_{i j}, \Delta p_{i j}, f_{i} \in L_{\infty}$, where $L_{\infty}$ is the space of essentially bounded functions on $[0,+\infty], \tau_{i j}$ and $\Delta \tau_{i j}:[0,+\infty] \rightarrow[0,+\infty]$ are measurable essentially bounded functions, $i, j=1, \ldots, n$. Concerning the operators $S_{i}$ we assume that $S_{i}: L_{\infty} \rightarrow L_{\infty}$ are linear continuous Volterra operators and the spectral radius $\rho\left(S_{i}\right)$ of the operator $S_{i}$ is less than 1 for $i=1, \ldots, n$. Our main purpose is to obtain conditions on the smallness of $\Delta p_{i j}(t)$ and $\Delta \tau_{i j}(t)$ such that the exponential stability of (2) is inherited by (1). Our second aim is to estimate the modulus of the difference of solutions to systems (1) and (2).

The studies of neutral functional differential equations have their own history. Equations in the form

$$
(x(t)-q(t) x(g(t)))^{\prime}+\sum_{i=1}^{m} b_{i}(t) x\left(h_{i}(t)\right)=f(t), \quad t \in[0, \infty),
$$

were considered in the well-known books [3, 24, 25] (see also the bibliography therein), where existence and uniqueness of solutions and especially stability and oscillation results for these equations were obtained. There exist problems in applications whose models can be written in the form [2]

$$
x^{\prime}(t)-q(t) x^{\prime}(g(t))+\sum_{i=1}^{m} b_{i}(t) x\left(h_{i}(t)\right)=f(t), \quad t \in[0, \infty) .
$$


This equation is a particular case of (1).

Let us note here that the operator $S_{i}: L_{\infty} \rightarrow L_{\infty}$ in (1) can be, for example, of the following forms:

$$
\begin{aligned}
& \left(S_{i} y\right)(t)=q_{i}(t) y\left(g_{i}(t)\right), \quad \text { where } g_{i}(t) \leq t, t \in[0, \infty), \\
& y(\xi)=0, \quad \text { for } \xi<0
\end{aligned}
$$

or

$$
\left(S_{i} y\right)(t)=\int_{0}^{t} k_{i}(t, s) y(s) d s, \quad t \in[0,+\infty)
$$

where $q_{j}(t)$ are essentially bounded measurable functions, $\tau_{j}(t)$ are measurable functions for $j=1, \ldots, m$, and $k_{i}(t, s)$ are summable with respect to $s$ and measurable essentially bounded with respect to $t$ for $i=1, \ldots, n$. All linear combinations of the operators (5) and (6) and their superpositions are also allowed.

The study of the neutral functional differential equations is essentially based on the questions of the action and estimates of the spectral radii of the operators in the spaces of discontinuous functions, for example, in the spaces of summable or essentially bounded functions. The operator (5) is a key object in this topic. Properties of this operator were studied, for example, in $[26,27]$. In order to achieve the action of the operator (5) in the space of essentially bounded functions $L_{\infty}$, we have to assume that mes $\left\{t: g_{i}(t)=c\right\}=0$ for every constant $c$. Let us suppose everywhere below that this condition is fulfilled. It is known that the spectral radius of the integral operator (6), considered on every finite interval $t \in[0, \omega]$, is equal to 0 (see, for example, [1]) and the inequality

$$
\sup _{t \geq 0} \int_{0}^{t}\left|k_{i}(t, s)\right| d s<1, \quad t \in[0, \infty), i=1, \ldots, n,
$$

implies that the spectral radii of the integral operators $S_{i}$ defined on the semiaxis are less than 1 . Concerning the operator (5), we can note that the inequality

$$
\underset{t \geq 0}{\operatorname{esssup}}\left|q_{i}(t)\right|<1, \quad t \in[0, \infty), i=1, \ldots, n,
$$

is a sufficient condition that the spectral radii $\rho\left(S_{i}\right)$ of the operators $S_{i}$ is less than 1 . Below we assume that this inequality is fulfilled.

Various results on existence and uniqueness of solutions to boundary value problems for (4) and its stability were obtained in [1], where also the basic results about the representation of solutions were presented. Note also in this connection the papers [28-31], where results on nonoscillation and positivity of Green's functions for neutral functional differential equations were obtained.

The goal of this paper is to obtain stability of uncertain systems and to estimate the difference between solutions of a 'real' system with uncertain coefficients and/or delays and corresponding 'model' system with fixed coefficients and delays. Instead of the traditional Lyapunov functionals technique, we propose an approach based on the idea of Azbelev's $W$-transform presented in the book [1]. This transform is a corresponding right regularization allowing researchers to reduce the analysis of boundary value problems to the study 
of systems of functional equations in the space of measurable essentially bounded functions. In corresponding cases estimates of norms of auxiliary linear operators (obtained as a result of $W$-transform) lead researchers to conclusions about existence, uniqueness, positivity and stability of solutions of given boundary value problems. This method works efficiently in the case when a 'model' used in $W$-transform is 'close' to a given 'real' uncertain system. In this paper we choose as a 'model', system for which we know solutions or estimates of the resolvent (Green's) operators. In this paper we demonstrate that systems with positive Cauchy operators [32, 33] represent a class of very convenient 'models'.

It should be noted that, although results are below formulated for the systems with neutral terms in the form (5), we can, using the approach of the papers [30, 31], obtain corresponding results with linear combinations and superpositions of the operators defined by (5) and (6).

\section{Stability and estimation of solution of neutral systems with uncertain coefficients}

Consider two systems

$$
\begin{aligned}
& x_{i}^{\prime}(t)-q_{i}(t) x_{i}^{\prime}\left(g_{i}(t)\right)+\sum_{j=1}^{n}\left(p_{i j}(t)-\Delta p_{i j}(t)\right) x_{j}\left(t-\tau_{i j}(t)\right)=f_{i}(t), \quad t \in[0, \infty), \\
& x_{i}^{\prime}(t)-q_{i}(t) x_{i}^{\prime}\left(g_{i}(t)\right)+\sum_{j=1}^{n} p_{i j}(t) x_{j}\left(t-\tau_{i j}(t)\right)=z_{i}(t), \quad t \in[0, \infty),
\end{aligned}
$$

where

$$
x_{i}(\xi)=x_{i}^{\prime}(\xi)=0, \quad i=1, \ldots, n, \xi<0 .
$$

Here $p_{i j}, \Delta p_{i j}, f_{i} \in L_{\infty}$, and $L_{\infty}$ is the space of essentially bounded functions on $[0, \infty]$. $\tau_{i j}:[0, \infty] \rightarrow[0, \infty]$ are measurable essentially bounded functions for $i, j=1, \ldots, n$.

It is known [1] that the general solution of (8) has the representation

$$
x_{i}(t)=\int_{0}^{t} \sum_{j=1}^{n} W_{i j}(t, s) z_{j}(s) d s+\sum_{j=1}^{n} W_{i j}^{0}(t, 0) x_{j}(0), \quad i=1, \ldots, n,
$$

where $W(t, s)=\left\{W_{i j}(t, s)\right\}_{i, j=1}^{n}$ is the Cauchy matrix of system $(8)$ and $W^{0}(t, 0)=\left\{W_{i j}^{0}(t, 0)\right\}$ is the fundamental matrix of system (8) satisfying the condition $W^{0}(0,0)=I$.

Substituting representation (9) into (7), we get

$$
\begin{gathered}
z_{i}(t)-\sum_{j=1}^{n} \Delta p_{i j}(t) \sigma\left(t-\tau_{i j}(t), 0\right) \int_{0}^{t-\tau_{i j}(t)} \sum_{k=1}^{n} W_{j k}\left(t-\tau_{i j}(t), s\right) z_{k}(s) d s \\
=\sum_{j=1}^{n} \Delta p_{i j}(t) \sigma\left(t-\tau_{i j}(t), 0\right) \sum_{k=1}^{n} W_{j k}^{0}\left(t-\tau_{i j}(t), 0\right) x_{k}(0)+f_{i}(t),
\end{gathered}
$$

where $\sigma(t, s)=\left\{\begin{array}{ll}1, & t \geq s, \\ 0, & t<s\end{array}\right.$ and $W_{j k}(\xi, s)=0$ if $\xi<s$.

We can write (10) in the vector form

$$
z(t)=\Omega z(t)+f^{*}(t)
$$


where the operator $\Omega: L_{\infty}^{n} \rightarrow L_{\infty}^{n}$ ( $L_{\infty}^{n}$ is the space of $n$ vector-functions with measurable essentially bounded components) is defined as follows:

$$
\Omega z(t)=\left\{\sum_{j=1}^{n} \Delta p_{i j}(t) \sigma\left(t-\tau_{i j}(t), 0\right) \int_{0}^{t-\tau_{i j}(t)} \sum_{k=1}^{n} W_{j k}\left(t-\tau_{i j}(t), s\right) z_{j}(s) d s\right\}_{i=1}^{n},
$$

and

$$
f^{*}(t)=\left\{f_{i}(t)+\sum_{j=1}^{n} \Delta p_{i j}(t) \sigma\left(t-\tau_{i j}(t), 0\right) \sum_{k=1}^{n} W_{j k}^{0}\left(t-\tau_{i j}(t), 0\right) x_{k}(0)\right\}_{i=1}^{n} .
$$

Estimating the norm of the operator $\Omega: L_{\infty}^{n} \rightarrow L_{\infty}^{n}$, we see that

$$
\|\Omega\| \leq \max _{1 \leq i \leq n} \operatorname{esssup}_{t \geq 0} \sum_{j=1}^{n}\left|\Delta p_{i j}(t)\right| \underset{t \geq 0}{\operatorname{esssup}} \int_{0}^{t} \sum_{k=1}^{n}\left|W_{j k}(t, s)\right| d s .
$$

Estimates of $\operatorname{esssup}_{t \geq 0} \int_{0}^{t}\left|W_{i j}(t, s)\right| d s$ is the key problem in our approach. Now describe the cases in which the Cauchy matrix $W(t, s)$ and the fundamental matrix $W^{0}(t, 0)$ of system (8) can be estimated.

Let us prove several assertions for the neutral system

$$
\begin{aligned}
& x_{i}^{\prime}(t)-q_{i}(t) x^{\prime}\left(g_{i}(t)\right)+\sum_{j=1}^{n} p_{i j}(t) x_{j}\left(t-\tau_{i j}(t)\right)=f_{i}(t), \quad t \in[0, \infty), i=1, \ldots, n, \\
& x_{i}(\xi)=x_{i}^{\prime}(\xi)=0, \quad i=1, \ldots, n, \xi<0 .
\end{aligned}
$$

Theorem 1 Let $p_{i i} \geq 0, q_{i} \geq 0$,

$$
\int_{h_{i}(t)}^{t} p_{i i}(s) d s \leq \frac{1}{e} \ln \frac{e}{1+\gamma_{i}(t) q_{i}(t)}, \quad t \in[0, \infty)
$$

where

$$
\begin{aligned}
& h_{i}(t)=\min \left\{t-\tau_{i i}(t), g_{i}(t)\right\}, \\
& \gamma_{i}(t)= \begin{cases}\frac{p_{i i}\left(g_{i}(t)\right)}{p_{i i}(t)} & \text { if } g_{i}(t) \geq 0, \\
0 & \text { if } g_{i}(t)<0,\end{cases}
\end{aligned}
$$

$p_{i j} \leq 0, i \neq j, i, j=1, \ldots, n$, then all elements $W_{i j}(t, s)$ of the Cauchy matrix and all elements $W_{i j}^{0}(t, 0)$ of the fundamental matrix of system (14) are nonnegative.

Proof According to the result of [31], the conditions of Theorem 1 imply the positivity of the Cauchy functions and fundamental solutions of the diagonal equations

$$
x_{i}^{\prime}(t)-q_{i}(t) x_{i}^{\prime}\left(g_{i}(t)\right)+p_{i i}(t) x_{i}\left(t-\tau_{i i}(t)\right)=f_{i}(t), \quad t \in[0, \infty), i=1, \ldots, n .
$$

Let us consider the system of diagonal equations denoting by $X(t, s)=\left\{X_{i j}(t, s)\right\}_{i, j=1}^{n}$ its Cauchy and $X^{0}(t, 0)=\left\{X_{i j}^{0}(t, 0)\right\}_{i, j=1}^{n}$ fundamental matrices, respectively. These matrices are 
the diagonal ones, i.e. $X_{i j}(t, s) \equiv 0$ and $X_{i j}^{0}(t, 0) \equiv 0$ for $j \neq i, i, j=1, \ldots, n$, and the elements $X_{i i}(t, s)$ and $X_{i i}^{0}(t, 0)$ coincide with the Cauchy functions and the fundamental solutions of the scalar diagonal equations, respectively. We can write the system

$$
\begin{aligned}
x_{i}(t)= & -\int_{0}^{t} X_{i i}(t, s) \sum_{j=1, j \neq i}^{n} p_{i j}(s) x_{j}\left(s-\tau_{i j}(s)\right) d s \\
& +\int_{0}^{t} X_{i i}(t, s) f_{i}(s) d s+X_{i i}^{0}(t, 0) x_{i}(0), \quad t \in[0, \infty), i=1, \ldots, n, \\
x_{i}(\xi)= & 0, \quad i=1, \ldots, n, \xi<0,
\end{aligned}
$$

which is equivalent to system (14). On every finite interval $[0, \omega]$ the spectral radius of the integral operator

$$
(K x)(t)=-\left\{\int_{0}^{t} X_{i i}(t, s) \sum_{j=1, j \neq i}^{n} p_{i j}(s) x_{j}\left(s-\tau_{i j}(s)\right) d s\right\}_{i, j=1}^{n}, \quad t \in[0, \omega],
$$

where

$$
x_{i}(\xi)=0, \quad i=1, \ldots, n, \xi<0
$$

is 0 . Denoting

$$
\phi(t)=\int_{0}^{t} X_{i i}(t, s) f_{i}(s) d s+X_{i i}^{0}(t, 0) x_{i}(0), \quad t \in[0, \infty), i=1, \ldots, n
$$

we can write (19) as $x(t)=\left((I-K)^{-1} \phi\right)(t)=\phi(t)+(K \phi)(t)+\left(K^{2} \phi\right)(t)+\cdots$ and consequently every component of solution vector $x(t)=\operatorname{col}\left\{x_{1}(t), \ldots, x_{n}(t)\right\}$ are nonnegative for nonnegative vectors $f=\operatorname{col}\left\{f_{1}, \ldots, f_{n}\right\}$ and $x(0)=\operatorname{col}\left\{x_{1}(0), \ldots, x_{n}(0)\right\}$. As $\omega$ tends to infinity we complete the proof of Theorem 1 .

Definition 1 The Cauchy matrix $W(t, s)$ of system (14) and its fundamental matrix $W^{0}(t, s)$ satisfies the exponential estimates, if there exist real numbers $N, \alpha>0$ such that $\left|W_{i j}(t, s)\right| \leq N e^{-\alpha(t-s)}$ and $\left|W_{i j}^{0}(t, 0)\right| \leq N e^{-\alpha t}$ for $0 \leq s \leq t<\infty, i, j=1, \ldots, n$.

Theorem 2 Let the conditions of Theorem 1 be fulfilled for system (14) and there exist $y_{i j} \geq 0$ for $i, j=1, \ldots, n$, such that $\sum_{j=1}^{n} y_{i j}>0$, and the system of inequalities

$$
\sum_{k=1}^{n} p_{i k}(t) y_{k j} \geq \delta_{i j}, \quad t \in[0, \infty), i=1, \ldots, n
$$

where $\delta_{i j}=\left\{\begin{array}{ll}1, & i=j, \\ 0, & i \neq j,\end{array}\right.$, be satisfied .

Then the Cauchy matrix $W(t, s)$ of system (14) and its fundamental matrix $W^{0}(t, 0)$ satisfy the exponential estimates and the integral estimate

$$
\int_{0}^{t} W_{i j}(t, s) d s \leq y_{i j} \quad \text { for } i, j=1, \ldots, n .
$$


Proof Let us extend the coefficients and delays in system (14) on the interval $[-H, 0)$, where $H=\max _{i, j=1, \ldots, n} \operatorname{esssup}_{t \geq 0} \tau_{i j}(t)$ as follows: $p_{i i}(t)=\operatorname{esssup}_{t \geq 0} p_{i i}(t), p_{i j}(t) \equiv 0$ for $i \neq j, \tau_{i j}(t) \equiv$ $0, q_{i}(t) \equiv 0$ for $i, j=1, \ldots, n, t \in[-H, 0)$, and consider system (14) on $[-H, \infty)$, calling it the extent system. The constant vector-function $Y_{j}(t) \equiv\left\{y_{i j}\right\}_{i=1}^{n}$ satisfies the inequality $x_{i}^{\prime}(t)-$ $q_{i}(t) x^{\prime}\left(g_{i}(t)\right)+\sum_{j=1}^{n} p_{i j}(t) x_{j}\left(t-\tau_{i j}(t)\right) \geq \delta_{i j}, t \in[-H, \infty), i=1, \ldots, n$. All entries of the Cauchy matrix of the extent system are nonnegative according to Theorem 1 . The Cauchy matrices of system (14) and the extent system coincide in $0 \leq s \leq t<\infty$. Using nonnegativity of all entries of the Cauchy matrix $W(t, s)$ of system (14) we get the inequalities $\int_{-H}^{t} W_{i j}(t, s) d s \leq$ $y_{i j}$ for $i, j=1, \ldots, n, t \in[-H, \infty)$ that implies inequalities (23). For every bounded right hand side, the solution of system (14) is bounded. From this the exponential estimates of entries of the Cauchy matrix $W(t, s)$ and the fundamental matrix $W^{0}(t, s)$ follow (see [1], Paragraph 5.3).

Analogously we can obtain the following assertion.

Theorem 3 Let the conditions of Theorem 1 be fulfilled for system (14), let there exist a vector $\gamma=\operatorname{col}\left\{\gamma_{1}, \ldots, \gamma_{n}\right\}$ such that $\gamma_{i}>0$ for $i=1, \ldots, n$, and

$$
\sum_{k=1}^{n} p_{i j}(t) \gamma_{j} \geq 1, \quad t \in[0, \infty), i=1, \ldots, n
$$

Then the Cauchy matrix $W(t, s)$ of system (14) and its fundamental matrix $W^{0}(t, 0)$ satisfy the exponential estimates and the integral estimate

$$
\int_{0}^{t} \sum_{j=1}^{n} W_{i j}(t, s) d s \leq \gamma_{i} \quad \text { for } i=1, \ldots, n \text {. }
$$

Theorem 4 Let all coefficients $p_{i j}$ be constants and the conditions of Theorem 1 be fulfilled for system (14). Then the Cauchy matrix $W(t, s)$ of system (14) and its fundamental matrix $W^{0}(t, 0)$ satisfy the exponential estimates if and only if all components of the solution vector $\gamma=\operatorname{col}\left\{\gamma_{1}, \ldots, \gamma_{n}\right\}$ of the algebraic system

$$
\sum_{k=1}^{n} p_{i j} \gamma_{j}=1, \quad i=1, \ldots, n
$$

are positive.

Proof Sufficiency follows from Theorem 3. To prove necessity we extend the coefficients and delays in system (14) on the interval $[-H, 0)$, where $H=\max _{i, j=1, \ldots, n} \operatorname{esssup}_{t \geq 0} \tau_{i j}(t)$ such that $\tau_{i j}(t) \equiv 0, q_{i}(t) \equiv 0, p_{i j}(t)=p_{i j}, f_{i}(t) \equiv 1$ for $t \in[-H, 0), i, j=1, \ldots, n$. The constant vector-function $\gamma=\operatorname{col}\left\{\gamma_{1}, \ldots, \gamma_{n}\right\}$ is now the solution of the system

$$
x_{i}^{\prime}(t)-q_{i}(t) x_{i}^{\prime}\left(g_{i}(t)\right)+\sum_{j=1}^{n} p_{i j} x_{j}\left(t-\tau_{i j}(t)\right)=1, \quad t \in[-H, \infty),
$$


satisfying the condition $x_{i}(-H)=\gamma_{i}, i=1, \ldots, n$. Using the formula of the solution's representation (see (9)) on $[-H, \infty)$, we obtain

$$
\gamma_{i}=\int_{-H}^{t} \sum_{j=1}^{n} W_{i j}(t, s) d s+\sum_{j=1}^{n} W_{i j}^{0}(t,-H) \gamma_{j}, \quad i=1, \ldots, n .
$$

All elements $W_{i j}(t, s)$ of the Cauchy matrix of system (27) are nonnegative. From the fact that $W_{i j}^{0}(t, 0)$ satisfies the exponential estimate we obtain $\gamma_{i}>0$ for $i=1, \ldots, n$.

Remark 1 Under the conditions of Theorem 4, the equalities

$$
\begin{aligned}
& \lim _{t \rightarrow \infty} \int_{0}^{t} \sum_{j=1}^{n} W_{i j}(t, s) d s=\gamma_{i} \quad \text { for } i=1, \ldots, n, \\
& \lim _{t \rightarrow \infty} \int_{0}^{t} W_{i j}(t, s) d s=y_{i j} \quad \text { for } i, j=1, \ldots, n,
\end{aligned}
$$

are true.

Theorem 5 Let $q_{i} \geq 0$, the inequality (15) be fulfilled and let there exist a vector $\gamma=$ $\operatorname{col}\left\{\gamma_{1}, \ldots, \gamma_{n}\right\}$ with all positive components such that

$$
p_{i i}(t) \gamma_{i}-\sum_{j=1, i \neq j}^{n}\left|p_{i j}(t)\right| \gamma_{j} \geq 1, \quad t \in[0, \infty), i=1, \ldots, n
$$

Then the Cauchy matrix $W(t, s)$ of system (14) and its fundamental matrix $W^{0}(t, 0)$ satisfy the exponential estimates and the integral estimates

$$
\begin{aligned}
& \int_{0}^{t} \sum_{j=1}^{n}\left|W_{i j}(t, s)\right| d s \leq \gamma_{i} \quad \text { for } i=1, \ldots, n, \\
& \int_{0}^{t}\left|W_{i j}(t, s)\right| d s \leq y_{i j} \quad \text { for } i, j=1, \ldots, n,
\end{aligned}
$$

where the constants $\gamma_{i}$ and $y_{i j}$ are defined by inequalities (31) and (22) (where there are $\left|p_{i k}(t)\right|$ instead of $\left.p_{i k}(t)\right)$, respectively.

Proof Consider the system

$$
\begin{aligned}
& x_{i}^{\prime}(t)-q_{i}(t) x_{i}^{\prime}\left(g_{i}(t)\right)+p_{i i}(t) x_{i}\left(t-\tau_{i i}(t)\right)-\sum_{j=1, i \neq j}^{n}\left|p_{i j}(t)\right| x_{j}\left(t-\tau_{i j}(t)\right)=f_{i}(t), \\
& \quad t \in[0, \infty),
\end{aligned}
$$

where

$$
x_{i}(\xi)=x_{i}^{\prime}(\xi)=0, \quad i=1, \ldots, n, \xi<0 .
$$


We can write the equivalent integral system

$$
\begin{aligned}
x_{i}(t)= & \int_{0}^{t} X_{i i}(t, s) \sum_{j=1, j \neq i}^{n}\left|p_{i j}(s)\right|(s) x_{j}\left(s-\tau_{i j}(s)\right) d s \\
& +\int_{0}^{t} X_{i i}(t, s) f_{i}(s) d s+X_{i i}^{0}(t, 0) x_{i}(0), \quad t \in[0, \infty), i=1, \ldots, n .
\end{aligned}
$$

The operator

$$
(|K| x)(t)=\left\{\int_{0}^{t} X_{i i}(t, s) \sum_{j=1, j \neq i}^{n}\left|p_{i j}(s)\right| x_{j}\left(s-\tau_{i j}(s)\right) d s\right\}_{i, j=1}^{n}, \quad t \in[0, \infty),
$$

where

$$
x_{i}(\xi)=0, \quad i=1, \ldots, n, \xi<0
$$

is positive. Condition (31) implies according to Theorem 5.6 [34] that its spectral radius $\rho(|K|)<1$. According to Theorem 5.3 [34] we obtain the inequality $\rho(K)<\rho(|K|)$ for the spectral radius of the operator $K$ defined by equality (20) for $t \in[0, \infty)$. The exponential estimates of $X_{i i}(t, s)$ and $X_{i i}^{0}(t, 0)$ imply that

$$
\varphi_{i}(t) \equiv \int_{0}^{t} X_{i i}(t, s) f_{i}(s) d s+X_{i i}^{0}(t, 0) x_{i}(0), \quad t \in[0, \infty), i=1, \ldots, n,
$$

is bounded for every bounded right hand sides $f(t)=\operatorname{col}\left\{f_{1}(t), \ldots, f_{n}(t)\right\}$. Comparing the operators $K$ and $|K|$ we see that $-(I-|K|)^{-1}\|\varphi\|=-\left(I+|K|+\left|K^{2}\right|+\cdots\right)\|\varphi\| \leq(I-K)^{-1} \varphi=$ $\left(I+K+K^{2}+\cdots\right) \varphi \leq\left(I+|K|+\left|K^{2}\right|+\cdots\right)\|\varphi\|=(I-|K|)^{-1}\|\varphi\|$ and consequently the solution vector $x(t)=\operatorname{col}\left\{x_{1}(t), \ldots, x_{n}(t)\right\}$ is bounded for every bounded $\varphi=\operatorname{col}\left\{\varphi_{1}(t), \ldots, \varphi_{n}(t)\right\}$. These inequalities prove the estimates (32) and (33). According to Theorem 3.5 [1], the Cauchy and fundamental matrices satisfy the exponential estimates.

Theorem 6 Let the conditions of Theorem 5 be fulfilled and the norm of the operator $\Omega$, defined by equality (12), satisfy the inequality $\|\Omega\|<1$, then the Cauchy matrix $W(t, s)$ of system (14) and its fundamental matrix $W^{0}(t, 0)$ satisfy the exponential estimates and the difference $\Delta x$ of solution $x$ of system (7) and $\bar{x}$ of system (14) satisfies the inequality

$$
\|\Delta x\| \leq \max _{1 \leq i \leq n} \frac{\|f\|}{1-\|\Omega\|} \gamma_{i}, \quad i=1, \ldots, n
$$

for sufficiently large $t$.

In order to prove Theorem 6 we can note that in the case $\|\Omega\|<1$ we get $\|z\| \leq \frac{1}{1-\|\Omega\|}\|f\|$. A reference to (9) completes the proof.

\section{Stability and estimation of solution of neutral systems with uncertain delays}

Consider the system

$$
x_{i}^{\prime}(t)-q_{i}(t) x_{i}^{\prime}\left(g_{i}(t)\right)+\sum_{j=1}^{n} p_{i j}(t) x_{j}\left(t-\theta_{i j}(t)\right)=f_{i}(t)
$$


Rewrite (39) in the form

$$
\begin{aligned}
& x_{i}^{\prime}(t)-q_{i}(t) x_{i}^{\prime}\left(g_{i}(t)\right)+\sum_{j=1}^{n} p_{i j}(t) x_{j}\left(t-\tau_{i j}(t)\right) \\
& +\sum_{j=1}^{n} p_{i j}(t)\left(x_{j}\left(t-\theta_{i j}(t)\right)-x_{j}\left(t-\tau_{i j}(t)\right)\right)=f_{i}(t) .
\end{aligned}
$$

We have

$$
z_{i}(t)+\sum_{j=1}^{n} p_{i j}(t)\left(x_{j}\left(t-\theta_{i j}(t)\right)-x_{j}\left(t-\tau_{i j}(t)\right)\right)=f_{i}(t)
$$

where

$$
x_{i}^{\prime}(t)-q_{i}(t) x_{i}^{\prime}\left(g_{i}(t)\right)+\sum_{j=1}^{n} p_{i j}(t) x_{j}\left(t-\tau_{i j}(t)\right)=z_{i}(t) .
$$

It is clear that the solution of (42) has the representation

$$
x_{i}(t)=\int_{0}^{t} \sum_{j=1}^{n} W_{i j}(t, s) z_{j}(s) d s+\sum_{j=1}^{n} W_{i j}^{0}(t, 0) x_{j}(0), \quad i=1, \ldots, n
$$

where $W(t, s)=\left\{W_{i j}(t, s)\right\}, i, j=1, \ldots, n$ is the Cauchy matrix of system $(42)$ and $W_{i j}^{0}(t, 0)$ is its fundamental matrix. Let us assume for simplicity that $t-\tau_{i j}(t) \geq 0$ and $t-\theta_{i j}(t) \geq 0$ for $t \geq 0$. If this is not fulfilled we can extend coefficients on the interval $[-H, 0)$ and consider the system on $[-H, \infty)$ as in the Section 2 .

It is clear that $x_{j}\left(t-\Theta_{i j}(t)\right)-x_{j}\left(t-\tau_{i j}(t)\right)=\int_{t-\Theta_{i j}(t)}^{t-\tau_{i j}(t)} x_{j}^{\prime}(s) d s$ and

$$
z_{i}(t)+\sum_{j=1}^{n} p_{i j}(t) \int_{t-\Theta_{i j}(t)}^{t-\tau_{i j}(t)} x_{j}^{\prime}(s) d s=f_{i}(t)
$$

From (42) we get $x_{i}^{\prime}(t)=z_{i}(t)-\sum_{j=1}^{n} p_{i j}(t) x_{j}\left(t-\tau_{i j}(t)\right)$. Substituting this into (43), we obtain

$$
z_{i}(t)=\sum_{j=1}^{n} p_{i j}(t) \int_{t-\Theta_{i j}(t)}^{t-\tau_{i j}(t)}\left(I-S_{j}\right)^{-1}\left(\sum_{k=1}^{n} p_{j k}(s) x_{j}\left(s-\tau_{j k}(s)\right)-z_{j}(s)\right) d s+f_{i}(t)
$$

where the operator $S_{j}$ is defined by (5), and after substituting $x$ from (43) we get

$$
\begin{aligned}
z_{i}(t)= & \sum_{j=1}^{n} p_{i j}(t) \int_{t-\Theta_{i j}(t)}^{t-\tau_{i j}(t)}\left(I-S_{j}\right)^{-1}\left(\sum _ { k = 1 } ^ { n } p _ { j k } ( s ) \left(\int_{0}^{s-\tau_{j k}(s)} \sum_{m=1}^{n} W_{k m}(s, \xi) z_{m}(\xi) d \xi\right.\right. \\
& \left.\left.+\sum_{m=1}^{n} W_{k m}^{0}\left(s-\tau_{k m}(s), 0\right) x_{m}(0)\right)-z_{j}(s)\right) d s+f_{i}(t) .
\end{aligned}
$$


Here the operator $\Omega: L_{\infty}^{n} \rightarrow L_{\infty}^{n}$, is defined as $\Omega=\operatorname{col}\left\{\Omega_{1}, \ldots, \Omega_{n}\right\}$, where every component $\Omega_{i}$ is defined as follows:

$$
\begin{aligned}
\left(\Omega_{i} z_{i}\right)(t)= & \sum_{j=1}^{n} p_{i j}(t) \int_{t-\Theta_{i j}(t)}^{t-\tau_{i j}(t)}\left(I-S_{j}\right)^{-1} \\
& \times\left(\sum_{k=1}^{n} p_{j k}(s)\left(\int_{0}^{s-\tau_{i k}(s)} \sum_{m=1}^{n} W_{k m}(s, \xi) z_{m}(\xi) d \xi\right) d s-z_{j}(s)\right) d s .
\end{aligned}
$$

We can write (46) in the vector form

$$
z(t)=\Omega z(t)+f(t)
$$

It is clear that

$$
\begin{aligned}
\|\Omega\| \leq & \max _{1 \leq 1 \leq n} \operatorname{esssup}_{t \geq 0} \sum_{j=1}^{n}\left|p_{i j}(t)\right| \frac{1}{1-q} \operatorname{esssup}\left\{\sum_{t \geq 0}^{n} p_{j k}(t) \gamma_{k}+1\right\} \\
& \times \underset{t \geq 0}{\operatorname{esssup}}\left|\theta_{i j}(t)-\tau_{i j}(t)\right|,
\end{aligned}
$$

where $q=\max _{1 \leq i \leq n} \operatorname{esssup}_{t \geq 0} q_{i}(t)$ and the components $\gamma_{i}$ of the vector $\gamma=\operatorname{col}\left\{\gamma_{1}, \ldots, \gamma_{n}\right\}$ satisfies the system of inequalities (31).

Theorem 7 Let the conditions of Theorem 5 be fulfilled and $\|\Omega\|<1$, then the Cauchy matrix $W(t, s)$ of system (39) and its fundamental matrix $W^{0}(t, 0)$ satisfy the exponential estimates and the difference $\Delta x$ of solution $x$ of system (39) and $\bar{x}$ of system (14) satisfies the estimate

$$
\|\Delta x\| \leq \max _{1 \leq i \leq n} \frac{\|f\|}{1-\|\Omega\|} \gamma_{i}, \quad i=1, \ldots, n,
$$

for sufficiently large $t$.

\section{Stability and estimation of solution of neutral systems with uncertain coefficients and delays}

Consider the system

$$
x_{i}^{\prime}(t)-q_{i}(t) x^{\prime}\left(g_{i}(t)\right)+\sum_{j=1}^{n}\left(p_{i j}(t)-\Delta p_{i j}(t)\right) x_{j}\left(t-\theta_{i j}(t)\right)=f_{i}(t)
$$

and the auxiliary system

$$
x_{i}^{\prime}(t)-q_{i}(t) x^{\prime}\left(g_{i}(t)\right)+\sum_{j=1}^{n} p_{i j}(t) x_{j}\left(t-\tau_{i j}(t)\right)=z_{i}(t) .
$$

The solution of (52) has the representation

$$
x_{i}(t)=\int_{0}^{t} \sum_{j=1}^{n} W_{i j}(t, s) z_{j}(s) d s+\sum_{j=1}^{n} W_{i j}^{0}(t, 0) x_{j}(0), \quad i=1, \ldots, n .
$$


Rewrite (51) in the form

$$
\begin{aligned}
x_{i}^{\prime}(t) & -q_{i}(t) x^{\prime}\left(g_{i}(t)\right)+\sum_{j=1}^{n}\left(p_{i j}(t)-\Delta p_{i j}(t)\right) x_{j}\left(t-\tau_{i j}(t)\right) \\
& -\sum_{j=1}^{n}\left(p_{i j}(t)-\Delta p_{i j}(t)\right) x_{j}\left(t-\tau_{i j}(t)\right)+\sum_{j=1}^{n}\left(p_{i j}(t)-\Delta p_{i j}(t)\right) x_{j}\left(t-\theta_{i j}(t)\right)=f_{i}(t) .
\end{aligned}
$$

Using the technique of two previous sections, we obtain the system of the following form:

$$
z(t)=\Omega z(t)+f^{*}(t)
$$

where $f(t)-f^{*}(t) \rightarrow 0$ when $t \rightarrow \infty$, and estimating the norm of the corresponding operator $\Omega$, we get

$$
\begin{aligned}
& \|\Omega\| \leq \max _{1 \leq i \leq n}\left\{\operatorname{esssup}_{t \geq 0} \sum_{j=1}^{n}\left|\Delta p_{i j}(t)\right| \gamma_{j}\right\} \\
& \quad+\max _{1 \leq i \leq n}\left\{\operatorname{esssup}_{t \geq 0} \sum_{j=1}^{n}\left(\left|p_{i j}(t)\right|+\left|\Delta p_{i j}(t)\right|\right)\right\} \frac{1}{1-q} \operatorname{esssup}\left|\theta_{i j}(t)-\tau_{i j}(t)\right| \\
& \quad \times \underset{t \geq 0}{\operatorname{esssup}}\left(\sum_{k=1}^{n}\left|p_{j k}(t)\right| \gamma_{k}+1\right),
\end{aligned}
$$

where $\gamma_{i}, i=1, \ldots, n$, are defined by inequalities (31).

Theorem 8 Let the conditions of Theorem 5 be fulfilled and $\|\Omega\|<1$, then the Cauchy matrix $C(t, s)$ of system (51) and its fundamental matrix $C^{0}(t, 0)$ satisfy the exponential estimates and the difference $\Delta x$ of solution $x$ of system (51) and $\bar{x}$ of system (14) satisfies the inequality

$$
\|\Delta x\| \leq \max _{1 \leq i \leq n} \frac{\|f\|}{1-\|\Omega\|} \gamma_{i}, \quad i=1, \ldots, n,
$$

for sufficiently large $t$.

\section{Competing interests}

The authors declare that they have no competing interests.

\section{Authors' contributions}

All authors worked on this paper together and have the same rights on the content of the paper. All authors read and approved the final manuscript.

\section{Author details}

'Department of Mathematics and Computer Science, Ariel University, Ariel, Israel. ${ }^{2}$ Department of Mathematical Modelling, Perm National Research Polytechnic University, Perm, Russia.

\section{Acknowledgements}

This paper was prepared as an open auxiliary theoretical result in the frame of the project 'Exploitation of a synergetic model for development of business of innovation type' on the topic No. 2013/276-C 'Development of a model of operation of innovation business as dynamic model with memory effect', supported by Perm National Research 
Polytechnic University with financial support of Ministry of Science and Education of Russian Federation (agreement No. 02.G25.31.0068 of 23.05.2013)

\section{Received: 16 December 2013 Accepted: 27 February 2014 Published: 12 Mar 2014}

\section{References}

1. Azbelev, NV, Maksimov, VP, Rakhmatullina, LF: Introduction to the Theory of Linear Functional-Differential Equations. Advanced Series in Mathematical Science and Engineering, vol. 3. World Federation Publishers Company, Atlanta (1995)

2. Kolmanovskii, V, Myshkis, AD: Introduction to Theory and Applications of Functional Differential Equations. Kluwer Academic, Dordrecht (1999)

3. Gopalsamy, K: Stability and Oscillation in Delay Differential Equations of Population Dynamics. Kluwer Academic, Dordrecht (1992)

4. Cao, Y, Sun, Y, Cheng, C: Delay-dependent robust stabilization of uncertain systems with multiple state delays. IEEE Trans. Autom. Control 43, 1608-1612 (1998)

5. Chen, XW, Liu, B: Existence and uniqueness theorem for uncertain differential equations. Fuzzy Optim. Decis. Mak. 9, 69-81 (2010)

6. Dong, Y, Liu, J: Exponential stabilization of uncertain nonlinear time-delay systems. Adv. Differ. Equ. 2012, Article ID 180 (2012). doi:10.1186/1687-1847-2012-180

7. Dong, Y, Mei, S, Wang, X: Novel stability criteria of nonlinear uncertain systems with time-varying delay. Abstr. Appl. Anal. 2011, Article ID 969674 (2011)

8. Dzhalladova, IA, Bastinec, J, Diblik, J, Khusainov, DY: Estimates of exponential stability for solutions of stochastic control systems with delay. Abstr. Appl. Anal. 2011, Article ID 920412 (2011)

9. Yao, K, Gao, JW, Gao, Y: Some stability theorems of uncertain differential equation. Fuzzy Optim. Decis. Mak. 12, 3-13 (2013)

10. Khusainov, D, Diblik, J, Bavstinec, J, Ryvolova, J: Interval stability of linear systems neutral type. Vyčisl. Prikl. Mat. (Kiev) 3(106), 148-160 (2011) (in Russian)

11. Kwon, OM, Park, JH: Exponential stability of uncertain dynamic systems including state delay. Appl. Math. Lett. 19, 901-907 (2006)

12. Kwon, $\mathrm{OM}$, Park, JH: Exponential stability analysis for uncertain neural networks with interval time-varying delays Appl. Math. Comput. 212, 530-541 (2009)

13. Phat, VN, Nam, PT: Exponential stability and stabilization of uncertain linear time-varying systems using parameter dependent Lyapunov function. Int. J. Control 80, 1333-1341 (2007)

14. Phat, VN, Niamsup, P: Stability analysis for a class of functional differential equations and applications. Nonlinear Anal., Theory Methods Appl. 71, 6265-6275 (2009)

15. Shao, H-Y: New delay-dependent stability criteria for systems with interval delay. Automatica 45, 744-749 (2009)

16. Shatyrko, AV, Khusainov, DY, Diblik, J, Bastinec, J, Rivolova, A: Estimates of perturbation of nonlinear indirect interval control system of neutral type. J. Autom. Inf. Sci. 43, 13-28 (2011)

17. Shatyrko, AV, Khusainov, DY: Absolute interval stability of indirect regulating systems of neutral type. J. Autom. Inf. Sci. 42, 43-54 (2010)

18. Shatyrko, AV, Khusainov, D: Interval stability investigations of different regulator systems with time delay by Lyapunov-Krasovskii functional. Bull. Taras Shevchenko Natl. Univ. Kyiv. Ser., Phys. Math. 3, $212-221$ (2009) (in Ukrainian)

19. Sun, YJ: Global stabilizability of uncertain systems with time-varying delays via dynamic observer-based output feedback. Linear Algebra Appl. 353, 91-105 (2002)

20. Tian, J, Zhou, X: Improved asymptotic stability criteria for neural networks with interval time-varying delay. Expert Syst. Appl. 37, 7521-7525 (2010)

21. Verriest, El, Florchinger, P: Stability of stochastic systems with uncertain time delays. Syst. Control Lett. 24, 41-47 (1995)

22. Yue, D: Robust stabilization of uncertain systems with unknown input delay. Automatica 40, 331-336 (2004)

23. Wang, TY, Xie, LH, De Souz, CE: Robust control of a class of uncertain nonlinear systems. Syst. Control Lett. 19, 139-149 (1992)

24. Erbe, LN, Kong, Q, Zhang, BG: Oscillation Theory for Functional Differential Equations. Dekker, New York (1995)

25. Gyori, I, Ladas, G: Oscillation Theory of Delay Differential Equations. Clarendon, Oxford (1991)

26. Drakhlin, ME: Operator of the internal superposition in the space of summable functions. Izv. Vyš̌. Učebn. Zaved., Mat. 5, 18-23 (1986)

27. Drakhlin, ME, Plyshevskaya, TK: To the theory of functional differential equations. Differ. Uravn. 14, 1347-1361 (1978)

28. Berezansky, L, Braverman, E: Non-oscillation properties of a linear neutral differential equations. Funct. Differ. Equ. 9(3-4), 275-288 (2002)

29. Berezansky, L, Braverman, E: Oscillation criteria for linear neutral differential equations. J. Math. Anal. Appl. 286 601-617 (2003)

30. Domoshnitsky, A: Extension of Sturm's theorem to equations with time-lag. Differ. Uravn. 19, 1475-1482 (1983)

31. Gusarenko, SA, Domoshnitskii, Al: Asymptotic and oscillation properties of the first order scalar functional-differential equations. Differ. Uravn. 25, 1480-1491 (1989)

32. Agarwal, RP, Berezansky, L, Braverman, E, Domoshnitsky, A: Nonoscillation Theory of Functional Differential Equations with Applications. Springer, New York (2012)

33. Domoshnitsky, A, Sheina, MV: Nonnegativity of Cauchy matrix and stability of systems with delay. Differ. Uravn. 25 201-208 (1989)

34. Krasnosel'skii, MA, Vainikko, GM, Zabreiko, PP, Rutitskii, JB, Stezenko, VJ: Approximate Methods for Solving Operator Equations. Nauka, Moscow (1969) (in Russian) 
10.1186/1687-2770-2014-55

Cite this article as: Domoshnitsky et al.: Stability and estimate of solution to uncertain neutral delay systems. Boundary Value Problems 2014, 2014:55

Submit your manuscript to a SpringerOpen ${ }^{\circ}$ journal and benefit from:

- Convenient online submission

- Rigorous peer review

- Immediate publication on acceptance

- Open access: articles freely available online

- High visibility within the field

- Retaining the copyright to your article

Submit your next manuscript at $\gg$ springeropen.com 